\title{
Freeze-dried primate sperm retains early reproductive potential after intracytoplasmic sperm injection
}

\begin{abstract}
Oocytes inseminated by intracytoplasmic sperm injection using fresh ejaculated or freeze-dried rhesus macaque sperm showed similar activation, sperm aster assembly, and male-female pronuclear apposition rates. (Fertil Steril ${ }^{\circledR} 2008 ; 89: 742-5$. (C2008 by American Society for Reproductive Medicine.)
\end{abstract}

Cryopreservation currently is used for the storage of sperm, embryos, and cells for agriculture, biomedical research, and gene banking. Storage of cryopreserved specimens in liquid nitrogen is, however, becoming increasingly undesirable because of safety issues $(1,2)$, risk of pathogen contamination (3), and high storage and transport costs (4). These issues emphasize the need for alternative safe and low-cost storage systems for biological specimens.

The generation of normal mouse pups from oocytes fertilized with freeze-dried (FD) sperm $(5,6)$ highlighted the potential of using drying as a preservation method for living cells. These findings were expanded to bovine (7), pig (8), rabbit (9), and rat (10). However, development to term was reported only in the two latter cases, and the sole kit was noted as dead at birth (9). Nevertheless, this procedure has never been reported in primates.

Although live births have occurred after rehydration of desiccated sperm, successful fertilization was obtained only after intracytoplasmic sperm injection (ICSI) (5-9), because the process subjects the cells to various levels of damage, and rehydrated sperm are immotile. Pregnancy rates vary, but the success of the process, especially in rodents, may be related to the fact that these species do not require the paternal contribution of the sperm centrosome for natural fertilization and embryonic development $(11,12)$. Hence, in mice, fertilization can be achieved after ICSI of badly damaged sperm, or even of just sperm heads (5). Development of techniques to store sperm in the dry state from species in which the paternal centrosome contribution is required for embryonic development, such as primates, together with assisted reproductive techniques such as ICSI, could offer safe and less expensive sperm banking.

Structures associated with sperm function, including the acrosome and mitochondria, which are damaged by freeze-

Received May 2, 2006; revised and accepted February 28, 2007. Supported by grants from the National Institutes of Health (Bethesda, Maryland; C.R.S.) as well as from Fundação para a Ciência e Tecnologia and Fundação Luso-Americana para o Desenvolvimento, Lisboa, Portugal (J.R.-S.).

Reprint requests: Luis Gabriel Sánchez-Partida, Ph.D., Monash Institute of Medical Research, Monash University, 27-31 Wright Street, Clayton, Victoria 3168, Australia (FAX: 61-3-95947416; E-mail: gabriel. sanchez-partida@med.monash.edu.au). drying protocols $(6,9)$, could serve as potential markers for the quality of different storage protocols. Mitochondria are considered to be vital for sperm survival (12), and recent studies have indicated an association between mitochondrial membrane potential and sperm motility (13).

Here, we present the first evidence that FD sperm from rhesus macaques retain some essential early oocyte activation and fertilization characteristics after ICSI after storage in the dark at room temperature for $\geq 1$ and $\leq 2$ months. After rehydration, plasma membrane (14), acrosomal contents (15), and mitochondrial integrity $(16,17)$ were assessed by using fluorescent probes, whereas centriole integrity was assessed by using electron microscopy (18). The functional ability of FD sperm was monitored by assessing oocyte activation (2nd polar body elicitation; female pronuclear formation) after ICSI (19-21), as well as microtubule aster formation, by using antitubulin immunocytochemistry $(20,21)$. Semen and oocytes were collected from mature pathogen-free rhesus males (3) and females (8), as described elsewhere (21-23). All animal procedures were approved by the Institutional Animal Care and Use Committee at the Oregon National Primate Research Center at Oregon Health and Science University (Beaverton, OR), where all the experimental work was performed.

Sperm were freeze-dried by diluting semen samples with a 0.3 M trehalose solution. Semen was then frozen as pellets on dry ice and was stored at $-196^{\circ} \mathrm{C}$ in liquid nitrogen for 24 hours before freeze-drying. Single pellets were transferred into borosilicate glass vials, allowed to dry for 12 hours, argon injected, and then sealed with a rubber stopper for storage in the dark at room temperature for $\leq 2$ months.

After rehydration, FD sperm were intact, with no obvious morphological defects in the head (Fig. 1A), but were clearly immotile. Plasma membrane integrity was compromised in all cells, as shown by the vital DNA marker stain, propidium iodide (Fig. 1B). Fresh and FD sperm were labeled by using the fluorescent lectin peanut agglutinin fluorescein isothiocyanate conjugate (23) to monitor the degree of cell disruption and the effects of freeze-drying on acrosomal contents. After $1 \%$ formaldehyde fixation and gentle permeabilization with $0.5 \%$ Triton X-100 detergent, peanut agglutinin fluorescein isothiocyanate conjugate labeled the acrosomal contents of both fresh (Fig. 1C) and FD sperm (Fig. 1D). This 


\section{FIGURE 1}
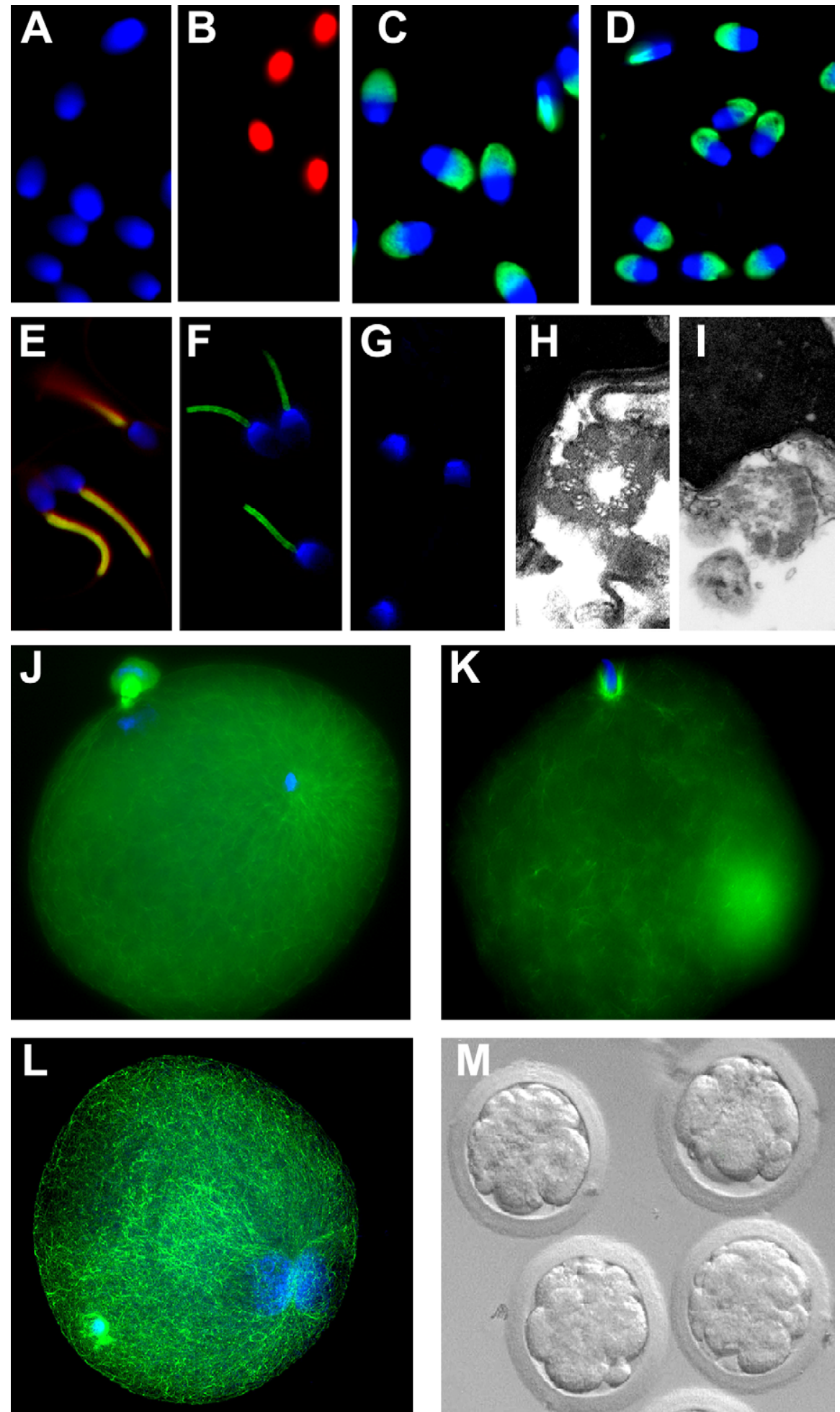

Sánchez-Partida. Freeze-dried sperm in primates. Fertil Steril 2008.

result confirms that sperm membrane integrity (both at the plasma membrane and acrosomal membrane levels) is compromised by freeze-drying but that acrosomal contents are retained on the sperm head. To further probe the effects of freeze-drying on sperm function, the mitochondrial membrane potential-sensitive dye JC-1 was used. The mid piece 
Organelle and functional integrity of freeze-dried rhesus sperm. Deoxyribonucleic acid of freeze-dried rhesus sperm labeled with (A) 6-diamino-2-phenylindole (DAPI, blue), showing no obvious morphological damage and with (B) propidium iodide $(r e d)$, indicating disrupted plasma membrane. Acrosomal contents of fresh ejaculated (C) and freeze-dried (D) sperm were visualized by using the fluorescent lectin peanut agglutinin fluorescein isothiocyanate conjugate (green) and DNA (DAPI, blue). (E-G) The vital probe JC-1 was used to assess mitochondrial activity. (E) In fresh ejaculated sperm, yellow-orange fluorescence, indicative of both high (red) and low (green) mitochondrial potential, was observed. In freeze-dried rhesus sperm, only low (green) mitochondrial membrane potential was visualized (F), whereas no mitochondrial activity was observed in fixed sperm $(\mathbf{G})$. By using electron microscopy, we could show that freeze-drying sperm in a $0.3 \mathrm{M}$ trehalose solution maintained the integrity of the proximal centrioles $(\mathbf{H})$; however, the proximal centrioles were damaged when sperm were freeze-dried in a Chatot-Biozek-Bavister (CBZ) medium (I). After ICSI, fresh $(\mathbf{J})$ and freeze-dried $(\mathbf{K}, \mathbf{L})$ sperm were able to nucleate microtubules (green) at the base of the sperm head, forming the sperm aster that directs pronuclear apposition. Blue, DNA. (M) Notwithstanding markers of cell death, rehydrated FD sperm retain some reproductive potency after ICSI, as assayed by embryonic development to the 8- to 16 -cell stage in vitro.

of fresh ejaculated sperm showed a typical pattern of both high (red) and low (green) mitochondrial membrane potential, indicating active mitochondria (Fig. 1E). In FD sperm, only green fluorescence was present (Fig. 1F), indicating compromised (i.e., low) mitochondrial function. Interestingly, however, no JC-1 label was detected in fixed cells (Fig. 1G).

A crucial aspect of sperm function relates to microtubule organization leading to pronuclear apposition and cell division after fertilization. Because the zygote's centrosome is derived from the blending of maternal constituent proteins (i.e., $\gamma$-tubulin) onto the incorporated sperm proximal centriole in primates (11), the status of this organelle after freeze-drying was evaluated. Electron microscopy showed similar intact proximal centrioles in fresh ejaculated sperm (18) and FD sperm in trehalose (Fig. 1H), but not when FD in Tyrode albumin lactate pyruvate (TALP) or ChatotZiomek-Bavister medium (CBZ) media (Fig. 1I). Freezedried sperm readily could activate oocytes after fertilization by ICSI, as in other species (24), thus suggesting that putative sperm-borne oocyte-activating factors are not affected by the process. Furthermore, microtubule asters emanating from the base of the decondensing sperm head (Fig. 1K) were similar to those in control fresh ejaculated sperm (25) (Fig. 1J) and could mediate pronuclear apposition (Fig. 1L), pointing to a functional sperm centriole in FD sperm. After ICSI, FD fertilization rates, as assessed by male and female pronuclear assembly, were comparable to those of fresh sperm (FD: 73.1\% [19/26]; fresh: 63.2\% [12/19]; 3 replicates). Zygotes produced with FD sperm and cultured in vitro cleaved at least once (19/19 FD inseminated vs. 9/12 produced with fresh ejaculated sperm), suggesting that oocyte activation by FD sperm was sufficient to complete the fertilization process and support early embryonic development. However, no development beyond the 8- to 16-cell stage (Fig. 1M) was observed in our limited data trials (4/12 embryos produced with FD sperm vs. 2/8 embryos produced with fresh ejaculated sperm), and no embryo transfers to establish pregnancy were attempted.

Until recently, attempts to dry mammalian cells have invariably caused irreversible damage. Live mice now have been generated after ICSI of sperm that had been FD and then stored for 3 months at room temperature (6). The process also has been applied in other species, although the rate of both embryo development and live births is much lower when compared with that in the mouse (7-10). We report here for the first time that FD sperm from primates that has been stored in the dark at room temperature for $\geq 1$ month can retain some early reproductive potential. Upon rehydration, sperm showed no motility and were considered moribund, as determined by propidium iodide staining. However, acrosomal contents were retained, sperm mitochondria showed some limited viability, and the proximal centriole remained intact, suggesting a limited effect of freeze-drying on primate sperm. Indeed, FD sperm retained the ability to initiate early development events after ICSI, including the following: [1] second polar-body elicitation; [2] female pronuclear formation; [3] reconstitution of a functional microtubule organizing center, the centrosome; [4] male sperm head decondensation and pronuclear formation; and [5] parental genome apposition within the activated oocyte cytoplasm. Freeze-dried sperm also could support some limited embryo development after ICSI. Primates, including humans, inherit their centrosomes from the sperm, unlike mice. After oocyte penetration, the spermborne centrosome then nucleates microtubules to form the sperm aster for pronuclear apposition and to provide the functional spindle poles at mitosis to ensure proper bipolar spindle assembly, all of which are key requirements for the successful conclusion of fertilization $(11,26)$. It has been suggested that the low developmental success obtained with FD sperm in nonrodent mammals may be related to damaged centrosomes (8). However, the rhesus sperm proximal centriole remained intact after freeze-drying and 
rehydration. Accordingly, after ICSI, the paternal centrosome was able to organize the sperm aster, and early embryo development was observed, similar to the development of oocytes that were inseminated with control ejaculated sperm. However, our limited data set precludes valid conclusions regarding potential blastocyst development rates or live offspring after embryo transfer to timed recipients.

Freeze-drying offers considerable potential for the future. It would avoid the complexities of current sperm-banking protocols and potentially could reduce costs considerably. It would further afford benefits for permanent banking of [1] special genetic material and/or cell lines, [2] donors in differing locations or those free of particular diseases, and [3] endangered species. The use of imperishable sperm, though, raises ethical issues that would need to be addressed before clinical use, and our results stress that any clinical speculations are premature. However, further research is clearly needed to develop protocols that would allow cells from different species to remain viable and fully functional after storage in a dry state for longer periods of time.

\section{Luis Gabriel Sánchez-Partida, Ph.D. ${ }^{\mathrm{a}}$ \\ Calvin R. Simerly, Ph.D. ${ }^{\mathrm{b}}$ \\ João Ramalho-Santos, Ph.D. ${ }^{\mathrm{b}, \mathrm{c}}$ \\ ${ }^{a}$ Monash Institute of Medical Research, Monash University, Clayton, Victoria, Australia; ${ }^{b}$ Department of Obstetrics, Gynecology, and Reproductive Sciences, Division of Regenerative and Developmental Medicine, University of Pittsburgh, Pittsburgh, Pennsylvania; and ${ }^{c}$ Center for Neuroscience and Cell Biology, Department of Zoology, University of Coimbra, Coimbra, Portugal}

\section{REFERENCES}

1. Tabata N, Funayama M, Ikeda T, Azumi J, Morita M. On an accident by liquid nitrogen-histological changes of skin in cold. Forensic Sci Int 1995;76:61-6.

2. Kernbach-Wighton G, Kijewski H, Schwanke P, Saur P, Sprung R. Clinical and morphological aspects of death due to liquid nitrogen. Int J Legal Med 1998;111:191-5.

3. Tedder RS, Zuckerman MA, Goldstone AH, Hawkins AE, Fielding A, Briggs EM, et al. Hepatitis B transmission from contaminated cryopreservation tank. Lancet 1995;346:137-40.

4. Tomlinson M, Sakkas D. Is a review of standard procedures for cryopreservation needed?: safe and effective cryopreservation-should sperm banks and fertility centres move toward storage in nitrogen vapour? Hum Reprod 2000;15:2460-3.

5. Ward MA, Kaneko T, Kusakabe H, Biggers JD, Whittingham DG, Yanagimachi R, et al. Long-term preservation of mouse spermatozoa after freeze-drying and freezing without cryoprotection. Biol Reprod 2003;69:2100-8.

6. Wakayama T, Yanagimachi R. Development of normal mice from oocytes injected with freeze-dried spermatozoa. Nat Biotechnol 1998;16: 639-41.

7. Keskintepe L, Pacholczyk G, Machnicka A, Norris K, Curuk MA, Khan I, et al. Bovine blastocyst development from oocytes injected with freeze-dried spermatozoa. Biol Reprod 2002;67:409-15.
8. Kwon IK, Park KE, Niwa K. Activation, pronuclear formation, and development in vitro of pig oocytes following intracytoplasmic injection of freeze-dried spermatozoa. Biol Reprod 2004;71:1430-6.

9. Liu JL, Kusakabe H, Chang CC, Suzuki H, Schmidt DW, Julian M, et al. Freeze-dried sperm fertilization leads to full-term development in rabbits. Biol Reprod 2004;70:1776-81.

10. Hirabayashi M, Kato M, Ito J, Hochi S. Viable rat offspring derived from oocytes intracytoplasmically injected with freeze-dried sperm heads. Zygote 2005;13:79-85.

11. Schatten G. The centrosome and its mode of inheritance: the reduction of the centrosome during gametogenesis and its restoration during fertilization. Dev Biol 1994;165:299-335.

12. Terada Y, Simerly CR, Hewitson L, Schatten G. Sperm aster formation and pronuclear decondensation during rabbit fertilization and development of a functional assay for human sperm. Biol Reprod 2000;62: 557-63.

13. St John JC, Sakkas D, Barratt CL. A role for mitochondrial DNA and sperm survival. J Androl 2000;21:189-99.

14. Garner DL, Johnson LA. Viability assessment of mammalian sperm using SYBR-14 and propidium iodide. Biol Reprod 1995;53:276-84.

15. Ramalho-Santos J, Moreno RD, Sutovsky P, Chan AW, Hewitson L, Wessel GM, et al. SNAREs in mammalian sperm: possible implications for fertilization. Dev Biol 2000;223:54-69.

16. Marchetti C, Jouy N, Leroy-Martin B, Defossez A, Formstecher P, Marchetti P. Comparison of four fluorochromes for the detection of the inner mitochondrial membrane potential in human spermatozoa and their correlation with sperm motility. Hum Reprod 2004;19: 2267-76.

17. St John JC, Ramalho-Santos J, Gray HL, Petrosko P, Rawe VY, Navara CS, et al. The expression of mitochondrial DNA transcription factors during early cardiomyocyte in vitro differentiation from human embryonic stem cells. Cloning Stem Cells 2005;7:141-53.

18. Manandhar G, Simerly C, Schatten G. Highly degenerated distal centrioles in rhesus and human spermatozoa. Hum Reprod 2000;15: 256-63.

19. Hewitson LC, Simerly CR, Tengowski MW, Sutovsky P, Navara CS, Haavisto AJ, et al. Microtubule and chromatin configurations during rhesus intracytoplasmic sperm injection: successes and failures. Biol Reprod 1996;55:271-80.

20. Hewitson L, Dominko T, Takahashi D, Martinovich C, RamalhoSantos J, Sutovsky P, et al. Unique checkpoints during the first cell cycle of fertilization after intracytoplasmic sperm injection in rhesus monkeys. Nat Med 1999;5:431-3.

21. Simerly C, Schatten G. Techniques for localization of specific molecules in oocytes and embryos. Methods Enzymol 1993;225:516-52.

22. Hewitson L, Takahashi D, Dominko T, Simerly C, Schatten G. Fertilization and embryo development to blastocysts after intracytoplasmic sperm injection in the rhesus monkey. Hum Reprod 1998;13:3449-55.

23. Sanchez-Partida LG, Maginnis G, Dominko T, Martinovich C, McVay B, Fanton J, et al. Live rhesus offspring by artificial insemination using fresh sperm and cryopreserved sperm. Biol Reprod 2000;63: 1092-7.

24. Liu QC, Chen TE, Huang XY, Sun FZ. Mammalian freeze-dried sperm can maintain their calcium oscillation-inducing ability when microinjected into mouse eggs. Biochem Biophys Res Commun 2005;328: 824-30.

25. Wu GJ, Simerly C, Zoran SS, Funte LR, Schatten G. Microtubule and chromatin dynamics during fertilization and early development in rhesus monkeys, and regulation by intracellular calcium ions. Biol Reprod 1996;55:260-70.

26. Simerly C, Wu GJ, Zoran S, Ord T, Rawlins R, Jones J, et al. The paternal inheritance of the centrosome, the cell's microtubule-organizing center, in humans, and the implications for infertility. Nat Med 1995;1: $47-52$. 